\title{
NEW CONSTRUCTIONS OF FUNCTION AND SET CHAINS
}

\author{
Martin Dowd \\ 1613 Wintergreen Pl. \\ Costa Mesa, CA 92626, USA
}

\begin{abstract}
Function and set chains can be constructed using ordinal notation systems. Here, earlier results are improved, and longer chains constructed. In particular, a simpler method is given for constructing set chains.
\end{abstract}

AMS Subject Classification: 03E55

Key Words: Galvin-Hajnal order, stationary reflection order

\section{Function Chains for Schemes}

Function chains for schemes have been given in [4]. These will be reviewed here, with some changes. Recall from [6] that a scheme over $\kappa$ where $\kappa \in$ Inac is a pair $\sigma=\langle\sigma, \phi\rangle$ where $\sigma<\kappa^{+}$and $\phi$ is a function whose domain is the set of limit ordinals $\alpha \leq \sigma$. For $\alpha \in \operatorname{Dom}(\phi), \phi(\alpha)$ is an increasing function with domain an ordinal $\eta \leq \kappa$, and whose range is an unbounded subset of $\alpha$. If $\operatorname{Cf}(\alpha)<\kappa$ then $\eta<\kappa$, and if $\operatorname{Cf}(\alpha)=\kappa$ then $\eta=\kappa$.

Recursions and inductions on schemes are broken into four cases, as follows.

Case 0: $\sigma=0$.

Case 1: $\sigma=\tau+1 . \boldsymbol{\tau}$ denotes $\boldsymbol{\sigma}_{\leq \tau}$.

Case 2: $\sigma \in \operatorname{Lim}, \operatorname{Cf}(\sigma)<\kappa$. For $\xi<\eta \sigma_{\xi}$ denotes $\phi(\sigma)(\xi)$; and $\boldsymbol{\sigma}_{\xi}$ denotes $\boldsymbol{\sigma}_{\leq \sigma_{\xi}}$

Case 3: $\sigma \in \operatorname{Lim}, \operatorname{Cf}(\sigma)=\kappa . \sigma_{\xi}$ and $\boldsymbol{\sigma}_{\xi}$ are as in case 2, for $\xi<\kappa$.

Received: August 15, 2013

(c) 2013 Academic Publications, Ltd. url: www.acadpubl.eu 
The thin subset $T_{\boldsymbol{\sigma}} \subseteq \kappa$ is defined recursively as follows.

$0: \emptyset$.

1: $T_{\tau}$.

2: $(\eta+1) \cup \cup_{\xi<\eta} T_{\sigma_{\xi}}$.

3: $\nabla \xi<\kappa T_{\sigma_{\xi}}$.

A function $f_{\sigma}: \operatorname{In}_{\kappa} \mapsto \kappa$ is defined recursively as follows.

0 : The identically 0 function.

1: $f_{\tau}+1$.

2: $\sup _{\xi<\eta} f_{\boldsymbol{\sigma}_{\xi}}$, with $f_{\boldsymbol{\sigma}}(\lambda)$ set to 0 if $\lambda \leq \theta$.

3: $\operatorname{dsup}_{\xi<\kappa} f_{\boldsymbol{\sigma}_{\xi}}$.

This definition was given in [4], for domain $\kappa$, and it was observed that these functions are the well-known canonical functions, which are unique mod the thin ideal. As noted in [7], the domain $\operatorname{In}_{\kappa}$ is convenient for defining $f_{\kappa^{+}}$. This results in the triviality that when $\lambda$ is the smallest inaccessible, $f_{\boldsymbol{\sigma}}$ is the empty function for all $\boldsymbol{\sigma}$. The proviso in case 2 ensures that $f_{\sigma}(\lambda)<\lambda^{+}$, as a simple induction shows; this does not seem to be necessary, though.

Lemma 1. Suppose $\boldsymbol{\sigma}^{\prime}, \boldsymbol{\sigma}$ are schemes, and $\lambda \notin T_{\boldsymbol{\sigma}^{\prime}} \cup T_{\boldsymbol{\sigma}}$.

a. If $\sigma^{\prime} \leq \sigma$ then $f_{\sigma^{\prime}}(\lambda) \leq f_{\sigma}(\lambda)$.

b. If $\sigma^{\prime}=\sigma$ then $f_{\sigma^{\prime}}(\lambda)=f_{\boldsymbol{\sigma}}(\lambda)$.

c. If $\sigma=\tau+1$ then $f_{\boldsymbol{\sigma}}(\lambda)=f_{\boldsymbol{\tau}}(\lambda)+1$.

d. If $\sigma^{\prime}<\sigma$ then $f_{\boldsymbol{\sigma}^{\prime}}(\lambda)<f_{\boldsymbol{\sigma}}(\lambda)$.

Proof. Part a follows by induction on $\sigma$. In case $0, \sigma$ is 0 also and the claim is trivial. In case 1 , if $\sigma^{\prime} \leq \tau$ then inductively $f_{\sigma^{\prime}}(\lambda) \leq f_{\tau}(\lambda)$, and clearly $f_{\boldsymbol{\tau}}(\lambda)<f_{\sigma}(\lambda)$. If $\sigma^{\prime}=\sigma$ then inductively $f_{\boldsymbol{\tau}^{\prime}}(\lambda)=f_{\boldsymbol{\tau}}(\lambda)$, and $f_{\boldsymbol{\sigma}^{\prime}}(\lambda)=f_{\boldsymbol{\sigma}}(\lambda)$ follows. In case 2, if $\sigma^{\prime}<\sigma$ then $\sigma^{\prime}<\sigma_{\xi}$ for some $\xi<\eta$. Inductively, $f_{\boldsymbol{\sigma}^{\prime}}(\lambda) \leq f_{\boldsymbol{\sigma}_{\xi}}(\lambda)$, and clearly $f_{\boldsymbol{\sigma}_{\xi}}(\lambda) \leq f_{\boldsymbol{\sigma}}(\lambda)$. If $\sigma^{\prime}=\sigma$, by the argument just given, $f_{\sigma_{\xi}^{\prime}}(\lambda) \leq f_{\boldsymbol{\sigma}}(\lambda)$ for all $\xi<\eta^{\prime}$, whence $f_{\sigma^{\prime}}(\lambda) \leq f_{\boldsymbol{\sigma}}(\lambda)$. The argument for case 3 is similar to that for case 2. Part b is immediate from part a. For part c let $\boldsymbol{\tau}_{1}=\boldsymbol{\sigma}_{\leq \tau}$ and use part b. Part d follows by parts a and c.

A scheme $\boldsymbol{\sigma} \downarrow \lambda$ over $\lambda$, for $\lambda \notin T_{\boldsymbol{\sigma}}$, may be defined recursively as follows. Write $\boldsymbol{\sigma} \downarrow \lambda$ as $\left\langle\sigma^{\prime}, \phi^{\prime}\right\rangle$.

0 : The scheme with $\sigma^{\prime}=0$.

1: $\boldsymbol{\tau} \downarrow \lambda$ with $\tau^{\prime}$ replaced by $\tau^{\prime}+1$.

2: $\sqcup_{\xi<\eta} \boldsymbol{\sigma}_{\xi} \downarrow \lambda$, with $\phi^{\prime}\left(\sigma^{\prime}\right)(\xi)$ set to $(\phi(\sigma)(\xi))^{\prime}$.

3: $\sqcup_{\xi<\lambda} \boldsymbol{\sigma}_{\xi} \downarrow \lambda$, with $\phi^{\prime}\left(\sigma^{\prime}\right)(\xi)$ set to $(\phi(\sigma)(\xi))^{\prime}$.

Lemma 2. Suppose $\boldsymbol{\sigma}$ is a scheme, $\lambda \in \operatorname{In}_{\kappa}$, and $\lambda \notin T_{\boldsymbol{\sigma}}$.

a, $f_{\boldsymbol{\sigma}}\left\lceil\lambda=f_{\boldsymbol{\sigma} \downarrow \lambda}\right.$. 
$b, f_{\sigma}(\lambda)=\sigma \downarrow \lambda$.

Proof. This is lemma 5 of [5]; for convenience the details are given. For part a, the cases of the induction are as follows, where $\mu \in \operatorname{In}_{\lambda}$.

$0: f_{0}(\mu)=0=f_{0 \downarrow \lambda}(\mu)$.

1: $f_{\boldsymbol{\sigma}}(\mu)=f_{\boldsymbol{\tau}}(\mu)+1=f_{\boldsymbol{\tau} \downarrow \lambda}(\mu)+1=f_{\boldsymbol{\sigma} \downarrow \lambda}(\mu)$.

2: $f_{\boldsymbol{\sigma}}(\mu)=\sup _{\xi<\eta} f_{\sigma_{\xi}}(\mu)=\sup _{\xi<\eta} f_{\sigma_{\xi \downarrow \lambda}}(\mu)=f_{\sigma \downarrow \lambda}(\mu)$.

3: $f_{\boldsymbol{\sigma}}(\mu)=\operatorname{dsup}_{\xi<\kappa} f_{\boldsymbol{\sigma}_{\xi}}(\mu)=\operatorname{dsup}_{\xi<\lambda} f_{\sigma_{\xi \downarrow \lambda}}(\mu)=f_{\boldsymbol{\sigma} \downarrow \lambda}(\mu)$.

For part $\mathrm{b}$, the cases of the induction are as follows.

0: $f_{0}(\lambda)=0=0 \downarrow \lambda$.

1: $f_{\boldsymbol{\sigma}}(\lambda)=f_{\boldsymbol{\tau}}(\lambda)+1=\tau \downarrow \lambda+1=\sigma \downarrow \lambda$.

2: $f_{\boldsymbol{\sigma}}(\lambda)=\sup _{\xi<\eta} f_{\boldsymbol{\sigma}_{\xi}}(\lambda)=\sup _{\xi<\eta} \sigma_{\xi} \downarrow \lambda=\sigma \downarrow \lambda$.

3: $f_{\boldsymbol{\sigma}}(\lambda)=\operatorname{dsup}_{\xi<\kappa} f_{\boldsymbol{\sigma}_{\xi}}(\lambda)=\operatorname{dsup}_{\xi<\lambda} \boldsymbol{\sigma}_{\xi} \downarrow \lambda=\sigma \downarrow \lambda$.

\section{Infinitary Veblen Functions}

The infinitary Veblen functions were defined in [11]. Further work on them includes [9] and [10]. A certain set of symbols for them are called Klammersymbols, or Schutte brackets. For convenience a self-contained treatment will be given here, following [11].

For basic facts about normal functions $f$ : Ord $\mapsto$ Ord, and club subclasses $R \subseteq$ Ord, see [8], section 4.2. In particular, the following hold.

- If $f$ is a normal function then $\operatorname{Ran}(f)$ is a club subclass.

- If $R$ is a club subclass then the function $\operatorname{Enum}(R)$ enumerating $R$ in natural order is normal.

- If $f$ is normal then the function $\operatorname{Fix}(f)$ enumerating the subclass $\{\alpha$ : $f(\alpha)=\alpha$ \} of fixed points of $f$ is normal.

- The intersection of a set of club class is club.

- Given a normal function $f$, suppose $f(\alpha)>\alpha$. Let $\alpha_{0}=\alpha$, and for $n \geq 0$ let $\alpha_{n+1}=f\left(\alpha_{n}\right)$. Then $\sup _{n} \alpha_{n}$ is the smallest fixed point of $f$ which is greater than $\alpha$.

- If $f(0)>0$ then $\operatorname{Fix}(f)(0)>f(0)$.

Let $\mathcal{A}$ be the set of ordinal valued sequences $\alpha_{0}, \ldots, \alpha_{\mu}$ for some $\mu$, where only finitely many $\alpha_{\xi}$ are nonzero, and $\alpha_{\mu}>0$ if $\mu>0$. $\mathcal{A}$ may be ordered in reverse lexicographic order, where if $\bar{\alpha}=\alpha_{0}, \ldots, \alpha_{\mu}$ and $\bar{\alpha}^{\prime}=\alpha_{0}^{\prime}, \ldots, \alpha_{\mu^{\prime}}^{\prime}$, then $\bar{\alpha}^{\prime}<_{\mathrm{rl}} \bar{\alpha}$ iff $\mu^{\prime}<\mu$, or $\mu^{\prime}=\mu$, and for some $\gamma \leq \mu, \alpha_{\gamma}^{\prime}<\alpha_{\gamma}$ and $\alpha_{\xi}^{\prime}=\alpha_{\xi}$ for $\gamma<\xi \leq \mu$. This relation is well-known to be a well-order (see [11] for example). 
Let $\mathcal{A}_{*}$ be the sequences obtained from the sequences of $\mathcal{A}$, by replacing some $\alpha_{\gamma}$ by $*$, provided $\alpha_{\delta}=0$ for $\delta<\gamma$. The order $<_{\text {rl }}$ may be extended to $\mathcal{A} \cup \mathcal{A}_{*}$, by taking $\alpha<*$ for $\alpha \in$ Ord.

Let $\theta$ be a cardinal. A function $\phi: \mathcal{A} \mapsto$ Ord will be defined, together with functions $\phi_{\tilde{\alpha}}$ : Ord $\mapsto$ Ord for each $\tilde{\alpha} \in \mathcal{A}_{*}$. The cases of the recursion are as follows.

1. $\phi_{\tilde{\alpha}}(\zeta)=\phi\left(S_{1}(\tilde{\alpha}, \zeta)\right)$, where in $S_{1}(\tilde{\alpha}, \zeta)$, * is replaced by $\zeta$ (and trailing 0's removed if $\tilde{\alpha}_{\mu}=*$ and $\zeta=0$ ).

2. For $\alpha \in \operatorname{Ord}, \phi(\alpha)=\theta^{\alpha}$.

3. For $\tilde{\alpha} \in \mathcal{A}_{*}$ with $\mu>0$, $\tilde{\alpha}_{0}=*$, $\tilde{\alpha}_{\gamma}=0$ for $0<\gamma<\nu$, and $\tilde{\alpha}_{\nu}>0$, $\phi_{\tilde{\alpha}}=\operatorname{Enum}\left(\cap_{\beta<\alpha_{\nu}, \gamma<\nu} \operatorname{Ran}\left(\operatorname{Fix}\left(\phi_{S_{2}(\alpha, \beta, \gamma)}\right)\right)\right)$, where in $S_{2}(\alpha, \beta, \gamma), \alpha_{0}$ is replaced by 0 and $\alpha_{\gamma}$ by $*$ (this is a null operation if $\gamma=0$ ), and $\alpha_{\nu}$ is replaced by $\beta$ (and trailing 0's removed if $\nu=\mu$ and $\beta=0$ ).

Lemma 3. For $\tilde{a} \in \mathcal{A}_{*}, \phi_{\tilde{\alpha}}$ is normal.

Proof. The proof is by induction on $<_{\mathrm{rl}}$. For the basis, $\tilde{\alpha}=*$; the lemma follows since $\zeta \mapsto \theta^{\zeta}$ is normal. For the induction step, if $\tilde{\alpha}_{0}=*$ the lemma follows by the induction hypothesis and the definition of $\phi$. Otherwise, using obvious notation write $\tilde{\alpha}$ as $0 \overline{0} * \bar{\eta}$; then $\phi_{0 \overline{0} * \bar{\eta}}(\zeta)=\phi_{* \overline{0} \zeta \bar{\eta}}(0)$. Suppose $\xi<\zeta$. Directly from the definition of $\phi, \operatorname{Ran}\left(\phi_{* \overline{0} \xi \bar{\eta}}\right) \supseteq \operatorname{Ran}\left(\phi_{* \overline{0} \zeta \bar{\eta}}\right)$ and $\phi_{* \overline{0} \xi \bar{\eta}}(0)<$ $\phi_{* \overline{0} \zeta \bar{\eta}}(0)$. In particular, $\phi_{0 \overline{0} * \bar{\eta}}(\xi)<\phi_{0 \overline{\bar{\eta}} * \bar{\eta}}(\zeta)$; that is, $\phi_{\tilde{\alpha}}$ is increasing.

For $\zeta \in \operatorname{Lim}$ let $\chi=\sup _{\xi<\zeta} \phi_{\tilde{\alpha}}(\xi)$. By what was just proved, $\chi \leq \phi_{\tilde{\alpha}}(\zeta)$, whence $\chi \leq \phi_{* \overline{0} \zeta \bar{\eta}}(0)$. Thus, to show that $\phi_{\tilde{\alpha}}$ is continuous it suffices to show that $\chi \in \operatorname{Ran}\left(\phi_{* \overline{0} \zeta \bar{\eta}}\right)$, since then $\chi=\phi_{* \overline{0} \zeta \bar{\eta}}(0)=\phi_{\tilde{\alpha}}(\zeta)$. For this in turn it suffices to show that $\phi_{S_{2}(* \overline{0} \zeta \bar{\eta}, \beta, \gamma)}(\chi)=\chi$ for $\beta<\zeta, \gamma<\nu$.

Suppose $\beta<\xi$, and for ease of notation let $\tilde{\alpha}_{1}$ denote $S_{2}(* \overline{0} \zeta \bar{\eta}, \beta, \gamma)$, and $\tilde{\alpha}_{2}$ denote $* \overline{0} \zeta \bar{\eta}$.

From the definition of $\phi, \operatorname{Ran}\left(\phi_{\tilde{\alpha}_{2}}\right) \subseteq \operatorname{Ran}\left(\operatorname{Fix}\left(\phi_{\tilde{\alpha}_{1}}\right)\right)$, and so $\phi_{\tilde{\alpha}_{2}}(0) \in \operatorname{Fix}\left(\phi_{\tilde{\alpha}_{1}}\right)$, whence $\phi_{\tilde{\alpha}_{1}}\left(\phi_{\tilde{\alpha}_{2}}(0)\right)=\phi_{\tilde{\alpha}_{2}}(0)$. Using this fact,

$$
\begin{aligned}
& \phi_{S_{2}\left(\phi_{* \overline{0} \zeta \bar{\eta}}, \beta, \gamma\right)}(\chi)= \\
& \phi_{S_{2}\left(\phi_{* \overline{0} \zeta \bar{\eta}}, \beta, \gamma\right)}\left(\sup _{\xi<\zeta} \phi_{\tilde{\alpha}}(\xi)\right)=\sup _{\xi<\zeta} \phi_{S_{2}\left(\phi_{* \overline{0} \zeta \bar{\eta}}, \beta, \gamma\right)}\left(\phi_{\tilde{\alpha}}(\xi)\right)= \\
& \sup _{\beta<\xi<\zeta} \phi_{S_{2}\left(\phi_{* \overline{0} \zeta \bar{\eta}}, \beta, \gamma\right)}\left(\phi_{\tilde{\alpha}}(\xi)\right)=\sup _{\beta<\xi<\zeta} \phi_{\tilde{\alpha}}(\xi)=\chi .
\end{aligned}
$$

Lemma 4. If $\tilde{\beta}<_{r l} \tilde{\alpha}$ then $\operatorname{Ran}\left(\operatorname{Fix}\left(\phi_{\tilde{\beta}}\right)\right) \cup\{1\} \supseteq \operatorname{Ran}\left(\phi_{\tilde{\alpha}}\right)$.

Proof. Writing $\tilde{\alpha}$ as $0 \overline{0} * \bar{\alpha}$., $\phi_{0 \overline{0} * \bar{\alpha}}(\zeta)=\phi_{* \overline{0} \zeta \bar{\alpha}}(0)$, provided $\zeta \neq 0$ if $\bar{\alpha}$ is null. By the definition of $\phi$ and induction on $<_{\mathrm{rl}}, \phi_{\tilde{\alpha}}(\zeta) \in \operatorname{Ran}\left(\operatorname{Fix}\left(\phi_{\tilde{\beta}}\right)\right) \cup\{1\}$, proving the lemma.

Let $\mathcal{A}_{*}^{0}=\left\{\tilde{\alpha} \in \mathcal{A}_{*}: \tilde{\alpha}_{0}=*\right\}$. The following are readily seen, for $\tilde{\beta}<_{\mathrm{rl}} \tilde{\alpha}$. 
- For $\tilde{\alpha} \in \mathcal{A}_{*}^{0}, 1 \in \operatorname{Ran}\left(\phi_{\tilde{\alpha}}\right)$ iff $\tilde{\alpha}=*$.

- For $\tilde{\alpha}, \tilde{\beta} \in \mathcal{A}_{*}^{0}$, If $\tilde{\beta}<_{\mathrm{rl}} \tilde{\alpha}$ then $\operatorname{Ran}\left(\operatorname{Fix}\left(\phi_{\tilde{\beta}}\right)\right) \supseteq \operatorname{Ran}\left(\phi_{\tilde{\alpha}}\right)$.

- For $\tilde{\alpha}, \tilde{\beta} \in \mathcal{A}_{*}, \operatorname{Ran}\left(\operatorname{Fix}\left(\phi_{\tilde{\beta}}\right)\right) \supseteq \operatorname{Ran}\left(\operatorname{Fix}\left(\phi_{\tilde{\alpha}}\right)\right)$.

For $\mu \in$ Ord let $\tilde{\alpha}_{\mu}$ be the element of $\mathcal{A}_{*}$ where $\tilde{\alpha}_{\mu 0}=*, \tilde{\alpha}_{\mu \mu}=1$, and $\tilde{\alpha}_{\mu \xi}=0$ for $0<\xi<\mu$. Let $\psi$ be the function $\mu \mapsto \tilde{\alpha}_{\mu}(0)$.

Lemma 5. The function $\psi$ is normal.

Proof. For $\mu \in$ Ord let $\tilde{\alpha}_{\mu}^{*}$ be the element of $\mathcal{A}_{*}$ where $\tilde{\alpha}_{\mu \mu}=*$ and $\tilde{\alpha}_{\mu \xi}=0$ for $0 \leq \xi<\mu$. From the definition of $\phi, \phi_{\tilde{\alpha}_{\mu}}=\operatorname{Enum}\left(\cap_{\gamma<\mu} \operatorname{Ran}\left(\operatorname{Fix}\left(\phi_{\tilde{\alpha}_{\gamma}^{*}}\right)\right)\right)$. It follows from this and lemma 4 that $\mu \mapsto \tilde{\alpha}_{\mu}(0)$ is increasing. For $\mu \in \operatorname{Lim}$ let $\chi=$ $\sup _{\gamma<\mu} \phi_{\tilde{\alpha}_{\gamma}}(0)$. From the definition of $\phi$, for $\gamma<\delta \operatorname{Ran}\left(\phi_{\tilde{\alpha}_{\delta}}\right) \subseteq \operatorname{Ran}\left(\operatorname{Fix}\left(\phi_{\tilde{\alpha}_{\gamma}^{*}}\right)\right)$. Using this fact, it follows by arguments as given above that $\phi_{\tilde{\alpha}_{\mu}}(0)=\chi$.

The least fixed point of $\psi$ is called the "large Veblen ordinal". Notation varies; $\Lambda_{0}$ will be used here. Clearly, $\Lambda_{0}=\phi_{\tilde{\alpha}_{\Lambda_{0}}}(0)=\sup _{\mu<\Lambda_{0}} \phi_{\tilde{\alpha}_{\mu}}(0)$. It follows that for $\mu<\Lambda_{0}, \Lambda_{0}$ is a fixed point of $\phi_{\tilde{\alpha}_{\mu}}$. Using lemma 4 , if $\tilde{\alpha}<\tilde{\alpha}_{\Lambda_{0}}$ then $\Lambda_{0}$ is a fixed point of $\phi_{\tilde{\alpha}}$, whence if $\zeta<\Lambda_{0}$ then $\phi_{\tilde{\alpha}}(\zeta)<\Lambda_{0}$.

Say that $\bar{\alpha} \in \mathcal{A}$ is maximal if $\phi(\bar{\beta}) \neq \phi(\bar{\alpha})$ whenever $\beta>_{\mathrm{rl}} \alpha$.

Lemma 6. For $\bar{\alpha} \in \mathcal{A}$, let $\nu_{1}>\cdots>\nu_{k}$ be the indices such that $\alpha_{\nu}>0$.

a. For $i>1, \phi(\bar{\alpha})>\alpha_{\nu_{i}}$.

b. $\phi(\bar{\alpha})>\alpha_{\nu_{1}}$ iff $\bar{\alpha}$ is maximal.

c. If $\phi(\bar{\alpha}) \notin \operatorname{Ran}(\psi)$ then $\nu_{k}<\phi(\bar{\alpha})$.

d. If $\tau \in \operatorname{Ran}\left(\phi_{*}\right)$ then $\tau=\phi(\bar{\alpha})$ for a unique maximal $\bar{\alpha}$.

Proof. For $1 \leq j<i$ let $\bar{\alpha}_{i}$ be $\bar{\alpha}$ with $\alpha_{\nu_{j}}$ replaced by 0 . It is easily seen that $\phi\left(\bar{\alpha}_{i-1}\right)>\phi\left(\bar{\alpha}_{i}\right) \geq \alpha_{\nu_{i}}$ for $i>1$, and part a follows. Write $\bar{\alpha}$ as $\overline{0} \alpha_{\nu} \alpha_{\nu+1} \bar{\alpha}^{\prime}$, where for ease of notation $\mu>\nu$ is assumed. Let $\tilde{\alpha}=\overline{0} * \alpha_{\nu+1} \bar{\alpha}^{\prime}$. Since $\phi_{\tilde{\alpha}}$ is normal, $\phi(\alpha) \geq \alpha_{\nu}$. Let $\bar{\beta}=* \overline{0} 0, \alpha_{\nu+1}+1, \bar{\alpha}^{\prime}$. Then $\operatorname{Ran}\left(\phi_{\tilde{\beta}}\right)=\operatorname{Ran}\left(\operatorname{Fix}\left(\phi_{\tilde{\alpha}}\right)\right)$, and so $\phi(\bar{\alpha})=\alpha_{\nu}$ iff $\alpha_{\nu} \in \operatorname{Ran}\left(\phi_{\tilde{\beta}}\right)$ iff $\bar{\alpha}$ is not maximal. The case $\nu=\mu$ is similar, proving part b. For part c, let $\gamma \in \operatorname{Ran}(\psi)$ be least such that $\phi(\bar{\alpha})<\gamma$. Then $\phi\left(\bar{\alpha}_{\nu_{k}}\right) \leq \phi(\bar{\alpha})<\gamma$, whence $\nu_{k}<\phi\left(\bar{\alpha}_{\nu_{k}}\right) \leq \phi(\bar{\alpha})$. For part $\mathrm{d}$, there is a $\mu$ such that $\tau<\phi_{\tilde{\alpha}_{\mu}}(0) \leq \phi_{\tilde{\alpha}_{\mu}}(\tau)$, and so there is a lexicographically least $\tilde{\alpha}$ such that $\tau<\phi_{\tilde{\alpha}}(\tau)$.

If $\tilde{\alpha}=*$ then $\tau=\phi(\zeta)$ for some $\zeta$ by hypothesis, and $\zeta<\tau$ since $\zeta=\tau$ is impossible. Now, $\tau=\phi_{\tilde{\beta}}(\tau)$ for $\tilde{\beta}<_{\mathrm{rl}} \tilde{\alpha}$. If $\tilde{\alpha}_{0}=*$ then by the definition of $\phi \tau=\phi_{\tilde{\alpha}}(\zeta)$ for some $\zeta$, and $\zeta=\tau$ is impossible. Otherwise $\tilde{\alpha}=0 \overline{0} * \bar{\alpha}^{\prime}$, and $\tau<\phi_{0 \overline{0} * \bar{\alpha}^{\prime}}(\tau)=\phi_{* \overline{0} \tau \bar{\alpha}^{\prime}}(0)$; but then by the definition of $\phi \tau=\phi_{* \overline{0} \tau \bar{\alpha}^{\prime}}(\zeta)$ for some $\zeta$, which is impossible. 
An infinitary Veblen function is a function $\phi_{k}\left(\zeta, \xi_{1}, \alpha_{1}, \ldots, \xi_{k}, \alpha_{k}\right)$ where $k \geq 1$. If $\xi_{1}<\cdots<\xi_{k}$ and $\alpha_{i}>0$ the arguments are said to be proper; the value of $\phi_{k}$ is $\phi(\bar{\alpha})$ where $\bar{\alpha}$ is the sequence $\zeta, \ldots \alpha_{1} \ldots \alpha_{2} \ldots \alpha_{k}$, where $\alpha_{i}$ occurs in position $1+\zeta_{i}$, and all other elements of $\bar{\alpha}$ are 0 . If the arguments are improper the value is 0 .

Corollary 7. For $\tau \in \operatorname{Ran}\left(\phi_{*}\right)$ with $\tau<\Lambda_{0}$ there are a unique $k$ and values $\zeta, \xi_{1}, \alpha_{1}, \ldots, \xi_{k}, \alpha_{k}<\tau$ such that $\tau=\phi_{k}\left(\zeta, \xi_{1}, \alpha_{1}, \ldots, \xi_{k}, \alpha_{k}\right)$.

Proof. Immediate.

Let $\mathrm{O}$ denote the function with ordinal arguments, where $\mathrm{O}\left(\alpha_{1}, \alpha_{2}\right)$ equals -1 if $\alpha_{1}<\alpha_{2}, 0$ if $\alpha_{1}=\alpha_{2}$, and +1 if $\alpha_{1}>\alpha_{2}$,

Lemma 8. Suppose $\tilde{\alpha}_{1}, \tilde{\alpha}_{2} \in \mathcal{A}_{*}^{0}$ and $\tilde{\alpha}_{1}<_{r l} \tilde{\alpha}_{2}$. Then $O\left(\phi_{\tilde{\alpha}_{1}}\left(\zeta_{1}\right)\right.$, $\left.\phi_{\tilde{\alpha}_{2}}\left(\zeta_{2}\right)\right)=O\left(\zeta_{1}, \phi_{\tilde{\alpha}_{2}}\left(\zeta_{2}\right)\right)$.

Proof. $\operatorname{Ran}\left(\phi_{\tilde{\alpha}_{2}}\right) \subseteq \operatorname{Ran}\left(\operatorname{Fix}\left(\phi_{\tilde{\alpha}_{1}}\right)\right)$, so $\phi_{\tilde{\alpha}_{1}}\left(\phi_{\tilde{\alpha}_{2}}\left(\zeta_{2}\right)\right)=\phi_{\tilde{\alpha}_{2}}\left(\zeta_{2}\right)$; the lemma follows.

\section{IV $_{o}$ Terms}

As in [7], a CNF function is a function $C_{k}\left(\eta_{k}, \sigma_{k}, \ldots, \eta_{1}, \sigma_{1}\right)$, where $k \geq 1$. If $\eta_{k}>\cdots>\eta_{1}$ and $0<\sigma_{i}<\theta$ the arguments are said to be proper, and the value of $C_{k}$ is $\theta^{\eta_{k}} \cdot \sigma_{k}+\cdots+\theta^{\eta_{1}} \cdot \sigma_{1}$. If the arguments are improper, the value is defined to be 0 .

Let $\mathrm{IV}_{o}$ be the system of terms, whose leaves are ordinals $\sigma$, and whose interior nodes are either a CNF function $C_{k}$ or an infinitary Veblen function $\phi_{k}$. A proper term is one where the arguments are proper at each interior node. A normal form term is a proper term where at each interior note, the value of the function is greater than the value of any of its arguments.

Theorem 9. If $\alpha<\Lambda_{0}$ then there is a unique normal form $I V_{o}$ term whose value is $\alpha$.

Proof. Let $\kappa^{+\eta_{k}} \cdot \sigma_{k}+\cdots+\kappa^{+\eta_{1}} \cdot \sigma_{1}$ be the Cantor normal form for $\alpha$. The proof is by induction on $\alpha$, with cases as follows, where $t$ denotes the term.

Case 0: $\alpha=0$. Then $t=0$.

Case 1: $k=1$ and $\eta_{1}=0$. Then $t=\sigma_{1}$. 


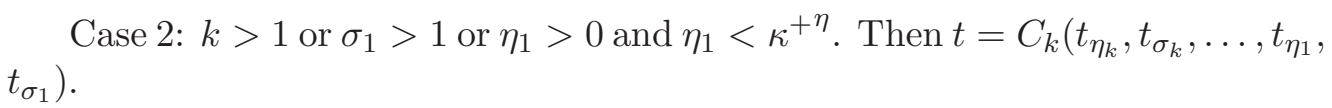

Case 3: $k=1$ and $\sigma_{1}=1$ and $\eta_{1}=\kappa^{+\eta}$. In this case, $t=\phi_{k}\left(t_{\zeta}, t_{\xi_{1}}, t_{\alpha_{1}}, \ldots\right.$, $\left.t_{\xi_{k}}, t_{\alpha_{k}}\right)$ where $\zeta, \xi_{1}, \alpha_{1}, \ldots, \xi_{k}, \alpha_{k}<\alpha$ are the unique values as in lemma 7 .

If $t_{i}$ is a term with value $\alpha_{i}$ for $i=1,2$, write $\mathrm{O}\left(t_{1}, t_{2}\right)$ for $\mathrm{O}\left(\alpha_{1}, \alpha_{2}\right)$. Let $\mathrm{P}(t)$ equal 1 if $t$ is proper, else 0 Let $\mathrm{N}(t)$ equal 1 if $t$ is normal, else 0 .

Lemma 10. a. $O\left(t_{1}, t_{2}\right)$ depends only on the values $O\left(\sigma_{1}, \sigma_{2}\right)$ and $P(\sigma)$ for the leaves of $t_{1}, t_{2}$.

b. $P(t)$ depends only on the values $O\left(\sigma_{1}, \sigma_{2}\right)$ and $P(\sigma)$ for the leaves of $t$.

c. $N(t)$ depends only on the values $O\left(\sigma_{1}, \sigma_{2}\right)$ and $P(\sigma)$ for the leaves of $t$.

Proof. Parts a and b are proved by induction on the total number of nodes. For part $\mathrm{b}$, if $t$ is a leaf then $\mathrm{P}(t)=1$. If $t$ is a CNF function, $\mathrm{P}(t)$ iff $\mathrm{P}(s)$ holds for all subterms $s$, and certain inequalities hold among the subterms. If $t$ is a IV function the argument is similar.

Part a falls into the following cases. If $t_{1}$ a leaf and $t_{2}$ a leaf then $\mathrm{O}\left(t_{1}, t_{2}\right)$ is known by hypothesis. Suppose $t_{1}$ an ordinal and $t_{2}$ is a CNF function. If $t_{2}$ is improper then $O\left(t_{1}, t_{2}\right)$ equals 0 if $t_{1}=0$, else 1 . If $t_{2}$ is proper then $O\left(t_{1}, t_{2}\right)$ equals -1 , unless $t_{2}=\kappa^{+0} \cdot \sigma_{2}$, in which case it equals $O\left(t_{1}, \sigma_{2}\right)$. Suppose $t_{1}$ an ordinal and $t_{2}$ is an IV function. If $t_{2}$ is improper then $O\left(t_{1}, t_{2}\right)$ equals 0 if $t_{1}=0$, else 1 . If $t_{2}$ is proper then $O\left(t_{1}, t_{2}\right)=-1$. In the remaining cases, if $t_{1}$ and $t_{2}$ are both improper then $O\left(t_{1}, t_{2}\right)=0$, if $t_{1}$ is improper and $t_{2}$ is proper then $O\left(t_{1}, t_{2}\right)=-1$, and if $t_{1}$ is proper and $t_{2}$ is improper then $O\left(t_{1}, t_{2}\right)=1$; so it may be assumed that $t_{1}$ and $t_{2}$ are proper. If $t_{1}$ and $t_{2}$ are both $\mathrm{CNF}$ functions, $O\left(t_{1}, t_{2}\right)$ is determined by the lexicographic order, using the induction hypothesis. Suppose $t_{1}$ is a CNF function and $t_{2}$ is an IV functions. If $\eta_{k}<\alpha_{2}$ (where $\eta_{k}$ ) is the leading exponent in $t_{1}$ ) then $\alpha_{1}<\alpha_{2}$. If $\eta_{k}>\alpha_{2}$ then $\alpha_{1}>\alpha_{2}$. If $\eta_{k}=\alpha_{2}$ then $\alpha_{1}>\alpha_{2}$, unless $k=1$ and $\sigma_{k}=1$, in which case $\alpha_{1}=\alpha_{2}$. If $t_{1}$ is an IV function and $t_{2}$ is a CNF functions, reverse the roles of $t_{1}$ and $t_{2}$ in the preceding argument. Suppose $t_{1}$ and $t_{2}$ are IV functions, say $\phi_{\tilde{\alpha}_{1}}\left(\zeta_{1}\right)$ and $\phi_{\tilde{\alpha}_{2}}\left(\zeta_{2}\right)$, If $\tilde{\alpha}_{1}<_{\mathrm{rl}} \tilde{\alpha}_{2}$ (which may be determined from the arguments using the induction hypothesis) then $\mathrm{O}\left(t_{1}, t_{2}\right)=\mathrm{O}\left(\zeta_{1}, \alpha_{2}\right)$. If $\tilde{\alpha}_{1}=\tilde{\alpha}_{2}$ then $\mathrm{O}\left(t_{1}, t_{2}\right)=\mathrm{O}\left(\zeta_{1}, \zeta_{2}\right)$. If $\tilde{\alpha}_{2}<_{\text {rl }} \tilde{\alpha}_{1}$ then $\mathrm{O}\left(t_{1}, t_{2}\right)=\mathrm{O}\left(\alpha_{1}, \zeta_{2}\right)$.

Part c now follows inductively. The claim is trivial if $t$ is a leaf. If $t$ is a CNF function then $t$ is normal if its subterms are normal, except if $k=1$ and $\eta_{k}=0$. If $t$ is an IV function then $t$ is normal if its subterms are normal, except if $t=\phi_{\tilde{\alpha}_{1}}\left(\phi_{\tilde{\alpha}_{2}}\left(\zeta_{2}\right)\right)$ where $\tilde{\alpha}_{1}<_{\mathrm{rl}} \tilde{\alpha}_{2}$. 


\section{IV Scheme Terms}

From hereon the cardinal $\theta$ will be $\kappa^{+}$for some $\kappa \in$ Inac. A scheme term over $\kappa$ is defined to be a term, whose leaves are 0 or a scheme over $\kappa$ with $\sigma>0$; and whose interior nodes are either a CNF function $C_{k}$ or an infinitary Veblen function $\phi_{k}$ where $k \geq 1$. The value $\alpha$ of a term $\boldsymbol{\alpha}$ is the value of the $\mathrm{IV}_{o}$ term obtained by replacing each scheme $\boldsymbol{\sigma}$ by its value $\sigma$. Clearly $\alpha$ depends only on the values of the schemes.

It follows from theorem 9 that for each $\alpha<\Lambda_{0}$ there is a normal form term $\boldsymbol{\alpha}$, whose ordinal is $\alpha$. $\boldsymbol{\alpha}$ is unique, up to the choice of schemes $\boldsymbol{\sigma}$ for the leaf ordinals $\sigma$. From hereon, unless specifically stated otherwise, IV scheme terms will be assumed to be in normal form.

For a term $\boldsymbol{\alpha}$ let $T_{\boldsymbol{\alpha}}$ be the union over the $\boldsymbol{\sigma}$ occurring at leaves of $\boldsymbol{\alpha}$ of the $T_{\boldsymbol{\sigma}}$.

Let $\boldsymbol{\alpha} \downarrow \lambda$ be $\boldsymbol{\alpha}$, with each leaf $\boldsymbol{\sigma}$ replaced by $\boldsymbol{\sigma} \downarrow \lambda$, each node $C_{k}$ replaced by $C_{\lambda k}$, and each node $\phi_{k}$ replaced by $\phi_{\lambda k}$, Given $\boldsymbol{\alpha}$, the notation $\alpha \downarrow \lambda$ will be used to denote the ordinal specified by $\boldsymbol{\alpha} \downarrow \lambda$.

Lemma 11. Suppose $\boldsymbol{\alpha}$ is a scheme, $\lambda \in \operatorname{In}_{\kappa}$, and $\lambda \notin T_{\boldsymbol{\alpha}}$.

a, $f_{\boldsymbol{\alpha}} \uparrow \lambda=f_{\boldsymbol{\alpha} \downarrow \lambda}$.

$b, f_{\boldsymbol{\alpha}}(\lambda)=\alpha \downarrow \lambda$.

Proof. For schemes this is lemma 2. Let the argument list of a function be denoted $A_{1}, \ldots, A_{l}$. For part a, suppose $\mu<\lambda$. For CNF functions, $f_{\boldsymbol{\alpha} \downarrow \lambda}(\mu)=$ $f_{C_{k}\left(A_{1} \downarrow \lambda, \ldots, A_{l} \downarrow \lambda\right)}(\mu)=C_{\mu k}\left(f_{A_{1} \downarrow \lambda}(\mu), \ldots, f_{A_{l} \downarrow \lambda}(\mu)\right)=C_{\mu k}\left(f_{A_{1}}(\mu), \ldots, f_{A_{l}}(\mu)\right)=$ $f_{C_{k}\left(A_{1}, \ldots, A_{l}\right)}(\mu)=f_{\boldsymbol{\alpha}}(\mu)$. The proof for IV functions is similar. For part $\mathrm{b}$, for CNF functions, $f_{\boldsymbol{\alpha}}(\lambda)=f_{C_{k}\left(A_{1}, \ldots, A_{l}\right)}(\lambda)=C_{\lambda k}\left(f_{A_{1}}(\lambda), \ldots, f_{A_{l}}(\lambda)\right)=C_{\lambda k}\left(A_{1} \downarrow\right.$ $\left.\lambda, \ldots, A_{l} \downarrow \lambda\right)=C_{k}\left(A_{1}, \ldots, A_{l}\right) \downarrow \lambda=\boldsymbol{\alpha} \downarrow \lambda$. The proof for IV functions is similar.

\section{Function Chains for IV Terms}

For each IV term $\boldsymbol{\alpha}$ a function $f_{\boldsymbol{\alpha}}: \operatorname{In}_{\kappa} \mapsto \kappa$ may be defined by recursion on $\boldsymbol{\alpha}$. The cases of the recursion are numbered as in theorem 9 , and are as follows. In cases 2 and $3, C_{\lambda k}$ and $\phi_{\lambda k}$ is written for the function over the cardinal $\lambda^{+}$.

0. $f_{0}=0$

1. $f_{\boldsymbol{\sigma}}$ where $\boldsymbol{\alpha}$ is the scheme $\boldsymbol{\sigma}$.

2. $f_{\boldsymbol{\alpha}}(\lambda)=C_{\lambda k}\left(f_{\boldsymbol{\eta}_{k}}(\lambda), f_{\boldsymbol{\sigma}_{k}}(\lambda), \ldots, f_{\boldsymbol{\eta}_{1}}(\lambda), f_{\boldsymbol{\sigma}_{1}}(\lambda)\right)$.

3. $f_{\boldsymbol{\alpha}}(\lambda)=\phi_{\lambda k}\left(f_{\boldsymbol{\zeta}}(\lambda), f_{\boldsymbol{\xi}_{1}}(\lambda), f_{\boldsymbol{\alpha}_{1}}(\lambda), \ldots, f_{\boldsymbol{\xi}_{k}}(\lambda), f_{\boldsymbol{\alpha}_{k}}(\lambda)\right)$. 
Theorem 12. Suppose $\boldsymbol{\alpha}$, etc. are proper terms, and $\lambda \in I_{\kappa}$.

a. If $\lambda \notin T_{\boldsymbol{\alpha}_{1}} \cup T_{\boldsymbol{\alpha}_{2}}$ then $O\left(f_{\boldsymbol{\alpha}_{1}}(\lambda), f_{\boldsymbol{\alpha}_{2}}(\lambda)\right)=O\left(\boldsymbol{\alpha}_{1}, \boldsymbol{\alpha}_{2}\right)$.

b. If $\lambda \notin T_{\boldsymbol{\alpha}}$ then $P\left(f_{\boldsymbol{\alpha}}(\lambda)\right)=P(\boldsymbol{\alpha})$.

c. If $\lambda \notin T_{\boldsymbol{\alpha}}$ then $N\left(f_{\boldsymbol{\alpha}}(\lambda)\right)=N(\boldsymbol{\alpha})$.

Proof. This follows by theorem 10 and lemma 1.

Theorem 13. Suppose $\boldsymbol{\alpha}$ and $\beta$ are $I V$ terms, $\alpha=\beta+1, \lambda \in \operatorname{In}_{\kappa}$, and $\lambda \notin T_{\alpha} \cup T_{\beta}$. Then $f_{\alpha}(\lambda)=f_{\beta}(\lambda)+1$.

Proof. In the CNF for $\alpha, \eta_{1}=0$; let $\boldsymbol{\alpha}_{p}$ be $\boldsymbol{\alpha}$ with the last term $\boldsymbol{\sigma}_{1}$ replaced by $\boldsymbol{\tau}_{1}$, or removed entirely if $\sigma_{1}=1$. By theorem $12, f_{\boldsymbol{\beta}}(\lambda)=T f_{\boldsymbol{\alpha}_{p}}(\lambda)$. It follows that $f_{\boldsymbol{\beta}}(\lambda)+1=T f_{\boldsymbol{\alpha}_{p}}(\lambda)+1=f_{\boldsymbol{\alpha}}(\lambda)$.

\section{Set Chains for IV Terms}

The reader is assumed to be familiar with [7]. A definition of $\mathrm{H}_{\boldsymbol{\alpha}}$ for an IV term $\boldsymbol{\alpha}$ will be given. A definition of $\mathrm{H}_{1 \boldsymbol{\alpha}}$ was given in [7] only for a subset of the BV terms. $\mathrm{H}$ is a map from subsets of $\mathrm{In}_{\kappa}$ to subsets of $\mathrm{In}_{\kappa}$; when it is necessary to specify $\kappa, \mathrm{H}_{\boldsymbol{\alpha}}$ will be denoted $\mathrm{H}_{\kappa \boldsymbol{\alpha}}$.

For an IV term $\boldsymbol{\alpha}$ and $X \subseteq \operatorname{In}_{\kappa}$, say that $\lambda \in \mathrm{H}_{\boldsymbol{\alpha}}(X)$ iff $\lambda \in X$ and $\mathrm{H}_{\boldsymbol{\beta}}(X \cap \lambda)$ is a stationary subset of $\lambda$ for all IV terms $\boldsymbol{\beta}$ over $\lambda$ where $\beta<f_{\boldsymbol{\alpha}}(\lambda)$.

For $X, Y \subseteq \operatorname{In}_{\kappa}$ say that $X \subseteq_{t} Y$ if $\left\{\lambda \in \operatorname{In}_{\kappa}: \lambda \in X\right.$ and $\left.\lambda \notin Y\right\}$ is thin; and similarly for $\equiv_{t}$.

Theorem 14. If $\alpha^{\prime} \leq \alpha$ then for any $X \subseteq I_{\kappa}, H_{\alpha^{\prime}}(X) \supseteq_{t} H_{\alpha}(X)$.

Proof. The proof is by induction on $\kappa$. For the basis, $\kappa$ is the smallest inaccessible cardinal, $X$ is always empty, and the claim is trivial. For arbitrary $\kappa$, there is a thin set $T$ such that if $\lambda \in \operatorname{In}_{\kappa}$ and $\lambda \notin T$ then $f_{\boldsymbol{\alpha}^{\prime}}(\lambda) \leq f_{\boldsymbol{\alpha}}(\lambda)$. For such $\lambda$, if $\mathrm{H}_{\boldsymbol{\beta}}(X \cap \lambda)$ is stationary for $\beta<f_{\boldsymbol{\alpha}}(\lambda)$, then by the induction hypothesis $\mathrm{H}_{\boldsymbol{\beta}}(X \cap \lambda)$ is stationary for $\beta<f_{\boldsymbol{\alpha}^{\prime}}(\lambda)$.

Lemma 15. Suppose $\boldsymbol{\alpha}$ is an $I V$ term, $X \subseteq \operatorname{In}_{\kappa}, \lambda \in \operatorname{In}_{\kappa}$, and $\lambda \notin T_{\boldsymbol{\alpha}}$. Then $H_{\boldsymbol{\alpha} \downarrow \lambda}(X \cap \lambda)=H_{\boldsymbol{\alpha}}(X) \cap \lambda$.

Proof. Suppose $\mu<\lambda$. Then $\mu \in \mathrm{H}_{\boldsymbol{\alpha} \downarrow \lambda}(X \cap \lambda)$ iff $\mathrm{H}_{\boldsymbol{\beta}}(X \cap \mu)$ is stationary for $\beta<f_{\boldsymbol{\alpha} \downarrow \lambda}(\mu)$ iff (by lemma 11) $\mathrm{H}_{\boldsymbol{\beta}}(X \cap \mu)$ is stationary for $\beta<f_{\boldsymbol{\alpha}}(\mu)$ iff $\mu \in \mathrm{H}_{\boldsymbol{\alpha}}(X)$. 
Theorem 16. Suppose $\alpha=\beta+1, \lambda \in \operatorname{In}_{\kappa}$, and $\lambda \notin T_{\boldsymbol{\alpha}}$. Then $\lambda \in H_{\boldsymbol{\alpha}}(X)$ iff $\lambda \in H\left(H_{\boldsymbol{\beta}}(X)\right)$.

Proof. $\lambda \in \mathrm{H}_{\alpha}(X)$ iff $\lambda \in X$ and $\mathrm{H}_{\gamma}(X \cap \lambda)$ is stationary for $\gamma$ with $\gamma<f_{\boldsymbol{\alpha}}(\lambda)$ iff $\lambda \in \mathrm{H}_{\boldsymbol{\beta}}(X)$ and (A) $\mathrm{H}_{\gamma}(X \cap \lambda)$ is stationary for $\gamma$ with $\gamma=f_{\boldsymbol{\alpha}}(\lambda)$. Using lemma 11.b, (A) holds iff $\mathrm{H}_{\boldsymbol{\beta} \downarrow \lambda}(X \cap \lambda)$ is stationary, and by lemma 15 this holds iff $\mathrm{H}_{\boldsymbol{\beta}}(X) \cap \lambda$ is stationary.

Recall from [7] the definition of $\rho_{R}$. By theorems 14 and 16, it follows that if $\mathrm{H}_{\boldsymbol{\alpha}}\left(\mathrm{In}_{\kappa}\right)$ is stationary then $\rho_{R}\left(\mathrm{H}_{\boldsymbol{\alpha}}\left(\mathrm{In}_{\kappa}\right) \geq \alpha\right.$. This was proved in [7] only for a subset of the BV terms (with a different definition of $\mathrm{H}_{\alpha}$; but see below).

\section{A Continuity Property}

As noted in [11], explicit ascending sequences can be given for limit ordinals less than $\Lambda_{0}$, using IV terms. A sequences $\boldsymbol{\alpha}_{\xi}$ for $\boldsymbol{\alpha}$ may be defined by recursion on $\boldsymbol{\alpha}$. Case 0 is irrelevant. In case 1 , let $\boldsymbol{\sigma}_{\xi}$ be as determined by the last node of $\sigma$.

Case 2 is divided into subcases as follows. In subcase 2.1, in the CNF for $\alpha, k>1$. Write $\boldsymbol{\alpha}=\boldsymbol{\alpha}_{1}+\boldsymbol{\alpha}_{2}$. Let $\boldsymbol{\alpha}_{\xi}$ equal $\boldsymbol{\alpha}_{1}+\boldsymbol{\alpha}_{2 \xi}$.

In subcase $2.2 \boldsymbol{\alpha}=\kappa^{+\boldsymbol{\eta}} \cdot \boldsymbol{\sigma}$ where $\sigma \in \operatorname{Lim}$ and $\sigma>1$. Let $\boldsymbol{\alpha}_{\xi}$ equal $\kappa^{+\boldsymbol{\eta}} \cdot \boldsymbol{\sigma}_{\xi}$.

In subcase $2.3 \boldsymbol{\alpha}=\kappa^{+\boldsymbol{\eta}}$ where $\eta \in \operatorname{Lim}$ and $\eta<\kappa^{+\eta}$. Let $\boldsymbol{\alpha}_{\xi}$ equal $\kappa^{+\boldsymbol{\eta}_{\xi}}$.

In subcase $2.4 \boldsymbol{\alpha}=\kappa^{+\boldsymbol{\eta}}$ where $\eta=\eta^{-}+1$. Let $\boldsymbol{\alpha}_{\xi}$ equal $\kappa^{+\boldsymbol{\eta}^{-}} \cdot \boldsymbol{\xi}$, where $\boldsymbol{\xi}$ is some particular scheme with ordinal $\xi$.

To divide case 3 into subcases, write the IV function as $\phi_{k}\left(\zeta, \pi_{1}, \alpha_{1}, \ldots\right)$. Cases will be denoted XYZ where $\mathrm{X}$ is the type of $\zeta$ ( $\mathrm{L}$ for limit, 0 , or $\mathrm{S}$ for successor), $\mathrm{Y}$ is the type of $\alpha_{1}$ ( $\mathrm{L}$ or $\left.\mathrm{S}\right)$, and $\mathrm{Z}$ is the type of $\xi_{1}(\mathrm{~L}, 0$, or $\mathrm{S})$; in some cases $\mathrm{Y}$ or $\mathrm{Z}$ may be ${ }^{*}$, denoting any possibility. If $\alpha$ is a successor ordinal write $\alpha^{-}$for its predecessor. Sequences $\boldsymbol{\alpha}_{\xi}$ in each case are as follows.

Case $\mathrm{L}^{* *}: \boldsymbol{\alpha}_{\xi}=\phi_{k}\left(\boldsymbol{\zeta}_{\xi}, \boldsymbol{\pi}_{1}, \boldsymbol{\alpha}_{1}, \ldots\right)$.

Case $0 L^{*}: \boldsymbol{\alpha}_{\xi}=\phi_{k}\left(0, \boldsymbol{\pi}_{1}, \boldsymbol{\alpha}_{1 \xi}, \ldots\right)$.

Case SL*: $\boldsymbol{\gamma}=\phi_{k}\left(\boldsymbol{\zeta}^{-}, \boldsymbol{\pi}_{1}, \boldsymbol{\alpha}_{1}, \ldots\right)+1, \boldsymbol{\alpha}_{\xi}=\phi_{k}\left(\boldsymbol{\gamma}, \boldsymbol{\pi}_{1}, \boldsymbol{\alpha}_{1 \xi} \ldots\right)$.

Case 0SL: $\boldsymbol{\alpha}_{\xi}=\phi_{k}\left(0, \boldsymbol{\pi}_{1 \xi}, \boldsymbol{\alpha}_{1}^{-}, \ldots\right)$.

Case SSL: $\boldsymbol{\gamma}=\phi_{k}\left(\boldsymbol{\zeta}^{-}, \boldsymbol{\pi}_{1}, \boldsymbol{\alpha}_{1}, \ldots\right)+1, \boldsymbol{\alpha}_{\xi}=\phi_{k}\left(\boldsymbol{\gamma}, \boldsymbol{\pi}_{1}, \boldsymbol{\alpha}_{1 \xi}, \ldots\right)$.

Case 0S0: $\boldsymbol{\alpha}_{0}=\phi_{k}\left(0,0, \boldsymbol{\alpha}_{1}^{-}, \ldots\right), \boldsymbol{\alpha}_{n+1}=\phi_{k}\left(\boldsymbol{\alpha}_{n}, 0, \boldsymbol{\alpha}_{1}^{-}, \ldots\right)$.

Case SS0: $\boldsymbol{\alpha}_{0}=\phi_{k}\left(\boldsymbol{\zeta}^{-}, 0, \boldsymbol{\alpha}_{1}, \ldots\right)+1, \boldsymbol{\alpha}_{n+1}=\phi_{k}\left(\boldsymbol{\alpha}_{n}, 0, \boldsymbol{\alpha}_{1}^{-}, \ldots\right)$. 
Case 0SS: $\boldsymbol{\alpha}_{0}=\phi_{k+1}\left(0, \boldsymbol{\pi}_{1}^{-}, 1, \boldsymbol{\pi}_{1}, \boldsymbol{\alpha}_{1}^{-}, \ldots\right), \boldsymbol{\alpha}_{n+1}=\phi_{k+1}\left(0, \boldsymbol{\pi}_{1}^{-}, \boldsymbol{\alpha}_{n}, \boldsymbol{\pi}_{1}, \boldsymbol{\alpha}_{1}^{-}\right.$,
$\ldots$. .

Case SSS: $\boldsymbol{\alpha}_{0}=\phi_{k}\left(\boldsymbol{\zeta}^{-}, \boldsymbol{\pi}_{1}, \boldsymbol{\alpha}_{1}, \ldots\right), \boldsymbol{\alpha}_{n+1}=\phi_{k+1}\left(0, \boldsymbol{\pi}_{1}^{-}, \boldsymbol{\alpha}_{n}, \boldsymbol{\pi}_{1}, \boldsymbol{\alpha}_{1}^{-}, \ldots\right)$.

Lemma 17. Suppose $\boldsymbol{\alpha}$ is a term, $\lambda \in \mathrm{In}_{\kappa}$, and $\lambda \notin T_{\boldsymbol{\alpha}}$. If $\alpha$ is a successor ordinal then $f_{\boldsymbol{\alpha}}(\lambda)$ is a successor ordinal. If $C f(\alpha)=\eta$ where $\eta<\kappa$ (whence $\eta<\lambda)$ then $C f\left(f_{\alpha}(\lambda)\right)=\eta$. If $C f(\alpha)=\kappa$ then $C f\left(f_{\boldsymbol{\alpha}}(\lambda)\right)=\kappa$. If $C f(\alpha)=\kappa^{+}$ then $C f\left(f_{\boldsymbol{\alpha}}(\lambda)\right)=\lambda^{+}$.

Proof. This follows by induction on $\alpha$, using the ascending sequences given above, and the definition of $f_{\boldsymbol{\alpha}}(\lambda)$.

The notation $f \leq_{t} g$ will be used to denote that $\{\lambda: f(\lambda)>g(\lambda\}$ is thin; and similarly for $=_{t}$. It is readily verified that sup and dsup produce least upper bounds in the order $\leq_{t}$. See also lemma 1.2 of [2]

Theorem 18. If $\eta<\kappa$ and $\alpha_{\xi}$ for $\xi<\eta$ is an ascending sequence with $\alpha=\sup _{\xi<\eta} \alpha_{\xi}$ then $f_{\boldsymbol{\alpha}}={ }_{t} \sup _{\xi<\eta} f_{\boldsymbol{\alpha}_{\xi}}$.

Proof. The proof is by induction on $\boldsymbol{\alpha}$. By remarks preceding the theorem it suffices to prove the claim for a particular sequence $\alpha_{\xi}$. The sequence used will be that given above. Case 0 is irrelevant. Case 1 follows directly. The remaining cases follow inductively, using properties of sup, with subcase 2.4 being irrelevant.

The theorem can be improved slightly. The set of $\lambda$ where the equation might not hold is contained in $T_{\boldsymbol{\alpha}} \cup(\eta+1) \cup \cup_{\xi<\eta} T_{\boldsymbol{\alpha}_{\xi}}$.

Theorem 19. If $\alpha_{\xi}$ for $\xi<\kappa$ is an ascending sequence with $\alpha=\sup _{\xi<\kappa} \alpha_{\xi}$ then $f_{\boldsymbol{\alpha}}={ }_{t} d \sup _{\xi<\kappa} f_{\boldsymbol{\alpha}_{\xi}}$.

Proof. The proof is by induction on $\boldsymbol{\alpha}$. As in the preceding theorem it suffices to prove the claim for the sequence given above. Case 0 is irrelevant. Case 1 follows directly. The remaining cases follow inductively, using the definition of dsup and properties of sup, with subcase 2.4 and the subcases of case 3 not involving an $\mathrm{L}$ being irrelevant.

Theorem 20. a. Suppose $\eta<\kappa, \alpha_{\xi}$ is ascending, and $\alpha=\sup _{\xi<\eta} \alpha_{\xi}$. Then $H_{\boldsymbol{\alpha}} \equiv_{t} \cap_{\xi<\eta} H_{\boldsymbol{\alpha}_{\xi}}$.

b. Suppose $\alpha_{\xi}$ is ascending and $\alpha=\sup _{\xi<\kappa} \alpha_{\xi}$. Then $H_{\boldsymbol{\alpha}} \equiv_{t}$ $B t_{\xi<\kappa} H_{\boldsymbol{\alpha}_{\xi}}$. 
Proof. For part a, $\lambda \in \mathrm{H}_{\boldsymbol{\alpha}}(X)$ iff $\mathrm{H}_{\boldsymbol{\beta}}(X \cap \lambda)$ is stationary whenever $\beta<$ $f_{\boldsymbol{\alpha}}(\lambda)$. By theorem 18, except for a thin set of $\lambda$, this holds iff, for all $\xi<\eta$, $\mathrm{H}_{\boldsymbol{\beta}}(X \cap \lambda)$ is stationary whenever $\beta<f_{\boldsymbol{\alpha}_{\xi}}(\lambda)$, iff $\lambda \in \mathrm{H}_{\boldsymbol{\alpha}_{\xi}}(X)$. Part b follows similarly, with " $\xi<\eta$ " replaced by " $\xi<\lambda$ ".

Readily from the definitions, $\mathrm{H}_{0}=\mathrm{Id}$ (the identity function). By theorem 16,18 , and $19, \mathrm{H}_{\boldsymbol{\sigma}}$ as defined here agrees with $\mathrm{H}^{\sigma}$ as defined in [7]. It may be seen that this continues to hold at $\kappa^{+}$; further discussion is omitted here.

\section{Enforceability}

Let $\Phi$ be the sentence $\forall A, C, X, Y, Z$ (" $A$ is an IV scheme term" $\wedge$ " $C$ is club" $\left.\wedge X=\operatorname{Inac} \wedge Y=\mathrm{H}_{A}(X) \wedge Z=Y \cap C \Rightarrow Z \neq \emptyset\right)$. Most of the subformulas of the matrix are readily seen to be $\Delta_{0}^{1}$; discussions will be given for the remaining ones.

There is a $\Delta_{0}^{1}$ predicate stating that the class $X$ represents a well-order on a subset of $\kappa$. This states that $X$ is a class of ordered pairs, which as a binary relation is transitive and reflexive, and has no descending chains of length $\omega$.

There is a $\Delta_{0}^{1}$ predicate stating that the class $X$ represents a scheme for $\kappa$. Namely, it represents a pair $\langle\sigma, \phi\rangle$ where $\sigma$ is represented as above, and $\phi$ is a function whose domain is the limit points $\alpha<\sigma$, where $\phi(\alpha)$ is a function with domain either an ordinal, or all ordinals, etc.

An IV term may be given as a class coding the sequence of classes $\left\langle t, \boldsymbol{\sigma}_{1}, \ldots\right.$, $\left.\boldsymbol{\sigma}_{k}\right\rangle$ where $t$ is a hereditarily finite set coding the tree of the term, and $\boldsymbol{\sigma}_{1}, \ldots, \boldsymbol{\sigma}_{k}$ are (codes for) the schemes at the leaves in order. It follows that the formula " $A$ is an IV scheme term" is $\Delta_{0}^{1}$.

A function $F$ : Ord $\mapsto$ Ord is a class (namely its ordered pairs). Let $f_{x}$ denote the value of $f_{\boldsymbol{\sigma}}$ at stage $x$ of the iteration. The class of triples $\left\langle x, \lambda, f_{x}(\lambda)\right\rangle$ may serve as a witness that $F=f_{A}$ for a scheme $A$. The predicate " $W$ is the witness to $F=f_{A}$ " is $\Delta_{0}^{1}$.

The predicate $\alpha=C_{\lambda k}\left(\eta_{1}, \sigma_{1}, \ldots, \eta_{k}, \sigma_{k}\right)$, in the values $\lambda, k,\left\langle\eta_{1}, \sigma_{1}, \ldots, \eta_{k}\right.$, $\left.\sigma_{k}\right\rangle$, is first-order definable, indeed no doubt $\Delta_{1}^{0}$. The same is true of the predicate $\alpha=\phi_{\lambda k}\left(\zeta, \xi_{1}, \alpha_{1}, \ldots, \xi_{k}, \alpha_{k}\right)$, Further discussion is omitted here.

The witness to $F=f_{A}$ where $A$ is any term is the sequence of classes $\left\langle F_{1}, \ldots, F_{r}, W_{1}, \ldots, W_{s}\right\rangle$ where $r$ is the number of nodes in the tree of $A, s$ is the number of leaves, for each leaf index $i W_{i}$ witnesses that $F_{l_{i}}=f_{\boldsymbol{\sigma}_{i}}$, and for each interior node index $i W_{i}$ witnesses that $F_{n_{i}}$ is obtained by applying the appropriate function per $\lambda$ to the $F_{j}$ of the sons. The predicate " $W$ is the witness to $F=f_{A}$ " is $\Delta_{0}^{1}$. 
From the foregoing the predicate $F=f_{A}$ is $\Sigma_{1}^{1}$, whence $\Delta_{1}^{1}$.

A term $\boldsymbol{\beta}$ over $\lambda \in \operatorname{In}_{\kappa}$ is a set. The predicates " $\beta$ is the ordinal of $\boldsymbol{\beta}$ " and " $\gamma=f_{\boldsymbol{\beta}}(\mu)$ " are first order definable. The function $\lambda \mapsto S_{\lambda}$ where $S_{\lambda}=$ $\left\{\langle\boldsymbol{\beta}, x, y\rangle: y=\mathrm{H}_{\boldsymbol{\beta}}(x)\right\}$ may be defined by recursion on $\lambda$; Indeed, $\langle\boldsymbol{\beta}, x, y\rangle \in S_{\lambda}$ iff $\forall \mu\left(\mu \in y\right.$ iff $\lambda \in x \wedge \forall \boldsymbol{\beta}\left(" \boldsymbol{\beta}\right.$ is term over $\mu$ " $\wedge \gamma<f_{\boldsymbol{\beta}}(\mu) \Rightarrow " \mathrm{H}_{\boldsymbol{\gamma}}(X \cap \mu)$ is stationary")).

Now, $Y=\mathrm{H}_{A}(X)$ may be expressed as $\forall \lambda(\lambda \in Y$ iff $\lambda \in X \wedge \forall \boldsymbol{\beta}$ (" $\boldsymbol{\beta}$ is term over $\lambda " \wedge \exists \gamma\left(\langle\lambda, \gamma\rangle \in F \wedge \beta<\gamma \Rightarrow " \mathrm{H}_{\boldsymbol{\beta}}(X \cap \lambda)\right.$ is stationary" $\left.)\right)$. From the foregoing, " $Y=\mathrm{H}_{A}(X)$ " is $\Delta_{1}^{1}$.

Say that an inaccessible cardinal $\kappa$ is $\Lambda_{0}$-Mahlo iff $\models_{V_{\kappa}} \Phi$.

Theorem 21. There is a $\Pi_{1}^{1}$ sentence $\Phi$ such that $\kappa$ is $\Lambda_{0}$-Mahlo iff $\models_{V_{\kappa}} \Phi$.

Proof. Immediate by remarks above.

Lemma 22. Suppose $\kappa$ is weakly compact. For each scheme $\boldsymbol{\sigma}$ there is a $\Pi_{1}^{1}$ formula $\Phi_{\boldsymbol{\sigma}}$ such that $\models_{V_{\kappa}} \Phi_{\boldsymbol{\sigma}}$, and for $\lambda \in I_{\kappa}$, if $\models_{V_{\lambda}} \Phi_{\boldsymbol{\sigma}}$ then $\boldsymbol{\sigma} \cap V_{\lambda}$ equals $\boldsymbol{\sigma} \downarrow \lambda$.

Proof. $\Phi_{\sigma}$ is constructed by recursion on $\sigma$. Various details are omitted, and may be found in [3]. Cases of the recursion are as follows, where $\Phi$ has second order parameters $\boldsymbol{\alpha}, P$. In case $0, \Phi$ is a sentence enforcing that $\kappa$ is inaccessible. In case $1, \Phi_{\sigma}$ is " $\sigma$ is a scheme" $\wedge$ " $\sigma$ is a successor ordinal" $\wedge$ $\forall \boldsymbol{\tau}\left(\tau=\boldsymbol{\sigma}^{-} \Rightarrow \Phi_{\boldsymbol{\sigma}^{-}}(\boldsymbol{\tau}, P)\right)$. In case $2, \Phi_{\boldsymbol{\sigma}}$ is " $\boldsymbol{\sigma}$ is a scheme" $\wedge \sigma \in \operatorname{Lim} \wedge \exists \eta($ " $\eta$ is the domain of the ascending sequence of the last node" $\wedge \forall \xi<\eta \forall \boldsymbol{\tau}(\tau=$ $\left.\boldsymbol{\sigma}_{\xi} \Rightarrow \Phi_{\boldsymbol{\sigma}_{\xi}}(\boldsymbol{\tau}, P)\right)$ ). The formulas $\Phi_{\boldsymbol{\sigma}_{\xi}}$ may be combined into a single formula in a well-known manner. Case 3 is similar.

Corollary 23. Suppose $\kappa$ is weakly compact. For each $I V$ term $\boldsymbol{\alpha}$ there is a $\Pi_{1}^{1}$ formula $\Phi_{\boldsymbol{\alpha}}$ such that $\models_{V_{\kappa}} \Phi_{\boldsymbol{\alpha}}$, and for $\lambda \in \operatorname{In}_{\kappa}$, if $\models_{V_{\lambda}} \Phi_{\boldsymbol{\alpha}}$ then $\boldsymbol{\alpha} \cap V_{\lambda}$ equals $\boldsymbol{\alpha} \downarrow \lambda$.

Proof. This follows readily from the lemma and the definition of $\boldsymbol{\alpha} \downarrow \lambda$.

Theorem 24. Suppose $\kappa$ is weakly compact. Then $\models_{V_{\kappa}} \Phi$.

Proof. It suffices to show by induction on $\boldsymbol{\alpha}$ that for all $\boldsymbol{\alpha}, \models_{V_{\kappa}}$ " $\mathrm{H}_{\boldsymbol{\alpha}}$ (Inac) is stationary". Inductively, $\models_{V_{\kappa}} \forall \boldsymbol{\beta}<\boldsymbol{\alpha}$ " $\mathrm{H}_{\boldsymbol{\beta}}$ (Inac) is stationary". By lemma 11 and corollary 23, for a stationary set of $\lambda, \models_{V_{\lambda}} \forall \boldsymbol{\beta}<f_{\boldsymbol{\alpha}}(\lambda)$ " $\mathrm{H}_{\boldsymbol{\beta}}$ (Inac) is stationary". $\models_{V_{\kappa}}$ "H $\mathrm{H}_{\boldsymbol{\alpha}}$ (Inac) is stationary" follows. 


\section{A New Axiom}

It seems helpful to define axiom $\mathrm{M}$ to be the second order axiom, to be added to NBG, which states that Ord is "...-Mahlo" where "..." is a specification of the length of stationary set chains which have been constructed. As of this paper, axiom M states that "Ord is $\Lambda_{0}$-Mahlo". The sentence $\Phi$ of the previous section gives a more detailed statement.

Only some brief remarks on justifying this axiom will be given here. The reader is assumed to be familiar with section 7 of [6]. Inductively, it may be assumed that $\forall \boldsymbol{\beta}<\boldsymbol{\alpha}$ " $\mathrm{H}_{\boldsymbol{\beta}}$ (Inac). Indeed, letting $\boldsymbol{\alpha}$ be a term in some sufficiently large outer universe, successive $\lambda$ such that $\models_{V_{\lambda}} \forall \boldsymbol{\beta}<f_{\boldsymbol{\alpha}}(\lambda)$ " $\mathrm{H}_{\boldsymbol{\beta}}(\operatorname{Inac})$ is stationary" may be collected. The general fact that something that may be repeated may be repeated stationarily often results in a universe in which " $\mathrm{H}_{\boldsymbol{\alpha}}$ (Inac) is stationary" holds.

As usual, more details of this justification would be desirable, but we omit this subject here.

\section{Normal Ultrafilters}

An application of function chains was given in [5], with further discussion given in [7]. An essential fact is the following.

Theorem 25. For any measurable cardinal $\kappa$, any normal ultrafilter $U$ on $\kappa$, and any $I V$ scheme term $\alpha$ over $\kappa, f_{\boldsymbol{\alpha}}$ represents $\alpha$ in the ultrapower $\operatorname{Ult}_{U}(V)$.

Proof. The proof is by induction on $\boldsymbol{\alpha}$, using Los' theorem. First the claim is proved for schemes. For case 0 the claim is immediate. For case 1, inductively $f_{\boldsymbol{\tau}}$ represents $\tau$, whence $f_{\boldsymbol{\tau}}+1$ represents $\tau+1$. For case 2 , inductively $f_{\boldsymbol{\sigma}_{\xi}}$ represents $\sigma_{\xi}$, whence $\sup _{\xi} f_{\boldsymbol{\sigma}_{\xi}}$ represents $\sup _{\xi} \sigma_{\xi}$. Case 3 is similar. For the induction on scheme terms, cases 0 and 1 have already been proved. Cases 2 and 3 follow readily by the definability of the functions $C_{k}$ and $\phi_{k}$.

It is independent whether there are canonical functions of rank $\kappa^{+}$(see [1]). The foregoing shows that the functions $f_{\boldsymbol{\alpha}}$ have a weaker property of interest. It would be of interest to obtain a characterization of the property which made no use of ultrafilters.

Suppose $\kappa$ is a measurable cardinal. Let $o(\kappa)$ denote its Mitchell order. For $\boldsymbol{\alpha}$ an IV scheme term let $S_{\boldsymbol{\alpha}}$ denote $\left\{\lambda \in \operatorname{In}_{\kappa}: o(\lambda) \geq f_{\boldsymbol{\alpha}}(\lambda)\right\}$. Recall the definition of $<_{R}$ from [7]. 
Theorem 26. Suppose $\alpha<o(\kappa)$. Then $S_{\boldsymbol{\alpha}}$ is stationary, and if $\beta<\alpha$ then $S_{\boldsymbol{\beta}}<_{R} S_{\boldsymbol{\alpha}}$.

Proof. Let $U_{1}$ be a normal ultrafilter on $\kappa$ with $O\left(U_{1}\right)=\alpha$. By lemma 19.34 of [Jech], $o$ represents $o\left(U_{1}\right)$ in $\operatorname{Ult}_{U_{1}}(V)$. Since by theorem $25 f_{\boldsymbol{\alpha}}$ also represents $\alpha=o\left(U_{1}\right),\left\{\lambda \in \operatorname{In}_{\kappa}: o(\lambda)=f_{\boldsymbol{\alpha}}(\lambda)\right\} \in U_{1}$. It follows that $S_{\boldsymbol{\alpha}}$ is stationary.

Suppose $\lambda \in S_{\boldsymbol{\beta}+1}$. Then $o(\lambda) \geq f_{\boldsymbol{\beta}+1}(\lambda)$, so except for a thin set of $\lambda$ there is a normal ultrafilter $U^{\prime}$ on $\lambda$ with $o\left(U^{\prime}\right)=f_{\boldsymbol{\beta}}(\lambda)$. By lemma 11 and theorem 25 , except for a thin set of $\lambda, f_{\boldsymbol{\beta}}\left\lceil\lambda\right.$ represents $f_{\boldsymbol{\beta}}(\lambda)$ in $\mathrm{Ult}_{U^{\prime}}(V)$, whence it represents $o\left(U^{\prime}\right)$. By an argument just given, $\left\{\mu<\lambda: o(\mu)=f_{\boldsymbol{\alpha}}(\mu)\right\} \in U^{\prime}$, whence $S_{\boldsymbol{\beta}} \cap \lambda \in U^{\prime}$. This shows that $S_{\boldsymbol{\beta}+1} \subseteq_{t} \mathrm{H}\left(S_{\boldsymbol{\beta}}\right)$, completing the proof of the theorem.

\section{References}

[1] J. Baumgartner, Ineffability properties of cardinals II, In: Logic, Foundations of Mathematics and Computability Theory (Ed-s: Butts and Hintikka), Springer, Netherlands (1977), 87-106, doi: 10.1007.

[2] O. Deiser, D. Donder, Canonical functions, non-regular ultrafilters and Ulam's problem on $\omega_{1}$, Journal of Symbolic Logic, 68 (2003), 713-739, doi: 10.2178 .

[3] M. Dowd, Iterating Mahlo's operation, Int. J. Pure Appl. Math., 9, No. 4 (2003), 469-512.

[4] M. Dowd, A lower bound on the Mahlo rank of a weakly compact cardinal, Int. J. Pure Appl. Math., 68, No. 4 (2011), 415-438.

[5] M. Dowd, Normal ultrafilters and Mahlo rank, Int. J. Pure Appl. Math., 68, No. 4 (2011), 487-491.

[6] M. Dowd, Improved results in scheme theory, Int. J. Pure Appl. Math., 76, No. 2 (2012), 173-190.

[7] M. Dowd, Scheme terms, Int. J. Pure Appl. Math., 81, No. 1 (2012), 111127.

[8] F. Drake, Set Theory, An Introduction to Large Cardinals, North Holland, England (1974). 
[9] K. Schutte, Kennzeichnung von Ordinalzahlen durch rekursiv definierte Funktionen, Math. Annalen, 127 (1954), 15-32.

[10] H. Simmons, Derivatives for ordinal functions and the Schutte brackets, preprint (2004), http://www.cs.man.ac.uk/ PAPERSandNOTES/Derivatives.dvi

[11] O. Veblen, Continuous increasing functions and transfinite ordinals, Trans. Amer. Math. Soc., 9 (1908), 280-292, doi: 10.1090. 